\title{
Molecular dynamics study of the longitudinal modes in disparate-mass binary liquid mixtures
}

\author{
N. Anento and J. A. Padró \\ Departament de Física Fonamental, Universitat de Barcelona, Diagonal, 647, 08028 Barcelona, Spain
}

(Received 12 January 2001; revised manuscript received 9 April 2001; published 20 July 2001)

\begin{abstract}
A series of molecular dynamics simulations of simple liquid binary mixtures of soft spheres with disparatemass particles were carried out to investigate the origin of the marked differences between the dynamic structure factors of some liquid binary mixtures such as the $\mathrm{Li}_{0.7} \mathrm{Mg}_{0.3}$ and $\mathrm{Li}_{0.8} \mathrm{~Pb}_{0.2}$ alloys. It is shown that the facility for observing peaks associated with fast-propagating modes in the partial Li-Li dynamic structure factor of $\mathrm{Li}_{0.8} \mathrm{~Pb}_{0.2}$ should be mainly attributed to the structure of this alloy, which is characterized by an incipient $A B A B$ ordering as found in molten salts. The longitudinal dispersion relations at intermediate wave vectors obtained from the longitudinal current spectra are very similar for the two alloys and reflect the existence of both fast-and slow-propagating modes of kinetic character associated with light and heavy particles, respectively. The influence of the hardness of the repulsive potential cores as well as the composition of the mixture on the longitudinal collective modes is also discussed.
\end{abstract}

DOI: 10.1103/PhysRevE.64.021202

PACS number(s): 61.20.Lc, 61.20.Ja, 05.20.Jj

\section{INTRODUCTION}

Studies of the collective dynamic properties in liquids at the atomic level are mostly based on the analysis of the dynamic structure factors and longitudinal current spectra, which are intimately related to the fluctuations in density. In recent years, the longitudinal collective modes in liquid alloys have been the subject of different experimental, theoretical, and computer-simulation analyses. Interest in this topic was stimulated by the results obtained by Bosse et al. [1] from a molecular dynamics (MD) simulation of the liquid $\mathrm{Li}_{0.8} \mathrm{~Pb}_{0.2}$ alloy. These authors observed the presence of noticeable peaks in the partial dynamic structure factor for the $\mathrm{Li}$ particles at intermediate wave vectors $(k)$ and frequencies $(\omega)$, i.e., within a $k-\omega$ interval between the kinetic and hydrodynamic regions. This interval can be suitably explored by both computer simulations and neutron-scattering experiments. The velocity corresponding to those modes was markedly higher than the sound velocity for $\mathrm{Li}_{0.8} \mathrm{~Pb}_{0.2}$ and they were called "fast sound" modes. Neutron-scattering experiments of the $\mathrm{Li}_{0.8} \mathrm{~Pb}_{0.2}$ and $\mathrm{Li}_{0.8} \mathrm{Tl}_{0.2}$ alloys [2] and binary mixtures of noble-gas fluids [3] also reflected the existence of these kinds of modes. Campa and Cohen [4] justified the existence of "fast sound" modes in binary mixtures of disparate-mass particles according to the kinetic theory. Recent MD simulations of the $\mathrm{Li}_{0.8} \mathrm{~Pb}_{0.2}$ [5] and $\mathrm{Li}_{0.7} \mathrm{Mg}_{0.3}$ [6] alloys suggested that the fast-propagating modes that were associated with the light particles are not soundlike modes but have a local kinetic character.

The partial intermediate scattering functions $\left[F^{i j}(k, t)\right]$ for distinct liquid binary alloys can show dramatic differences. This is evident if we observe the $F^{i j}(k, t)$ functions for $\mathrm{Li}_{0.8} \mathrm{~Pb}_{0.2}$ and $\mathrm{Li}_{0.7} \mathrm{Mg}_{0.3}$ in Fig. 1, which were calculated by MD simulation using the potential by Copestake et al. [7] and a potential obtained by the neutral pseudoatom method (NPA) [8,9], respectively. It should be noted that the $\mathrm{Li}_{0.8} \mathrm{~Pb}_{0.2}$ alloy has a marked ionic character, whereas $\mathrm{Li}_{0.7} \mathrm{Mg}_{0.3}$ may be considered as representative of an ideal simple liquid binary mixture. The static structure factors obtained from MD simulations of the $\mathrm{Li}_{0.8} \mathrm{~Pb}_{0.2}$ alloy using the potential by Copestake et al. [7] are in qualitative accord with the results from diffraction experiments while there is good agreement between the dynamic properties obtained from both MD and experiments $[5,10]$. The NPA potential reproduces rather well the total static structure factor for the $\mathrm{Li}_{0.7} \mathrm{Mg}_{0.3}$ alloy [9]. The $F^{i j}(k, t)$ 's for the $\mathrm{Li}_{0.8} \mathrm{~Pb}_{0.2}$ alloy show a fast-decaying oscillatory behavior that is similar to that usually shown by the $F(k, t)$ functions for onecomponent liquids. Then the $F^{i j}(k, t)$ 's for $\mathrm{Li}_{0.8} \mathrm{~Pb}_{0.2}$ can be Fourier-transformed to obtain the corresponding partial dynamic structure factors $\left[S^{i j}(k, \omega)\right]$. The $S^{\operatorname{LiLi}}(k, \omega)$ functions for the different $k$ 's show a noticeable peak that was associated with fast-propagating modes. On the contrary, the $F^{i j}(k, t)$ 's for $\mathrm{Li}_{0.7} \mathrm{Mg}_{0.3}$ show much larger values than for $\mathrm{Li}_{0.8} \mathrm{~Pb}_{0.2}$ and decay very slowly without any noticeable oscillation. The large discrepancies between the $F^{i j}(k, t)$ results for $\mathrm{Li}_{0.8} \mathrm{~Pb}_{0.2}$ and $\mathrm{Li}_{0.7} \mathrm{Mg}_{0.3}$ could be attributed to the bigger mass difference between particles of the two species in the former alloy. However, recent MD results for isotopic alloys [6] showed that the discrepancies should not be associated with the differences in the mass of particles but rather to other characteristics such as the differences in the interatomic potentials.

The main objective of this study is to investigate the origin of the discrepancies in the longitudinal collective dynamic properties of $\mathrm{Li}_{0.8} \mathrm{~Pb}_{0.2}$-like and $\mathrm{Li}_{0.7} \mathrm{Mg}_{0.3}$-like mix-

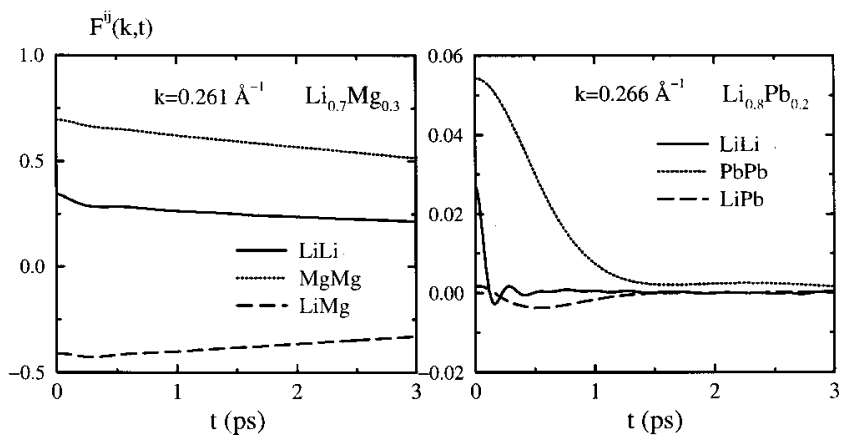

FIG. 1. Partial intermediate scattering functions for the $\mathrm{Li}_{0.8} \mathrm{~Pb}_{0.2}$ and $\mathrm{Li}_{0.7} \mathrm{Mg}_{0.3}$ alloys. 
TABLE I. Density, composition and interaction potentials of the different simulated systems. SS are soft-sphere potentials $V(r)$ $=4 \varepsilon(\sigma / r)^{6}$; LJ are ordinary LJ potentials; RLJ are repulsive LJ potentials (LJ truncated at its minimum). In all cases $\varepsilon=119.8 k$ and $T$ $=120 \mathrm{~K}$.

\begin{tabular}{|c|c|c|c|c|c|c|c|c|}
\hline & $\begin{array}{c}\text { System } \\
A\end{array}$ & $\begin{array}{c}\text { System } \\
A 1\end{array}$ & $\begin{array}{c}\text { System } \\
B\end{array}$ & $\begin{array}{c}\text { System } \\
C\end{array}$ & $\begin{array}{c}\text { System } \\
D\end{array}$ & $\begin{array}{c}\text { System } \\
D 1\end{array}$ & $\begin{array}{c}\text { System } \\
E\end{array}$ & $\begin{array}{c}\text { System } \\
F\end{array}$ \\
\hline $\begin{array}{c}\text { Density } \\
\left(\AA^{-3}\right)\end{array}$ & $2.4 \times 10^{-2}$ & $2.4 \times 10^{-2}$ & $2.4 \times 10^{-2}$ & $2.76 \times 10^{-2}$ & $3.4 \times 10^{-2}$ & $3.4 \times 10^{-2}$ & $2.4 \times 10^{-2}$ & $2.4 \times 10^{-2}$ \\
\hline $\begin{array}{l}\text { Molar } \\
\text { fraction }\end{array}$ & $x_{1}=0.75$ & $x_{1}=0.75$ & $x_{1}=0.75$ & $x_{1}=0.75$ & $x_{1}=0.75$ & $x_{1}=0.75$ & $x_{1}=0.25$ & $x_{1}=0.25$ \\
\hline$V_{11}(r)$ & SS & RLJ & SS & SS & SS & RLJ & SS & SS \\
\hline$\sigma_{11}(\AA)$ & 3.405 & 3.405 & 3.405 & 2.96 & 2.96 & 2.96 & 3.405 & 3.405 \\
\hline$V_{12}(r)$ & SS & RLJ & $\mathrm{LJ}$ & $\mathrm{LJ}$ & $\mathrm{LJ}$ & $\mathrm{LJ}$ & SS & $\mathrm{LJ}$ \\
\hline$\sigma_{12}(\AA)$ & 3.405 & 3.405 & 3.405 & 3.405 & 2.37 & 2.37 & 3.405 & 3.405 \\
\hline$V_{22}(r)$ & SS & RLJ & SS & SS & SS & RLJ & SS & SS \\
\hline$\sigma_{22}(\AA)$ & 3.405 & 3.405 & 3.405 & 3.85 & 3.85 & 3.85 & 3.405 & 3.405 \\
\hline
\end{tabular}

tures at intermediate wave vectors, i.e., between the hydrodynamic and the kinetic regimes. One of the advantages of the MD technique is that it allows us to perform computer-simulation experiments specially designed to check the influence of a given factor on the behavior of the system. The influence of the mass difference between particles on the longitudinal modes was recently discussed [6]. In this paper, we analyze the $F^{i j}(k, t)$ functions and corresponding spectra resulting from MD simulations of simple liquid binary mixtures of soft spheres with the same mass ratio but different interaction potential functions. The potentials were sketched to model the most significant features of the interactions in simple liquid mixtures and partially ionic alloys, respectively. Liquid mixtures in which the heavy particles are in the majority have also been simulated and the results compared with those obtained for analogous systems in which the heavy particles are in the minority. Earlier MD results on simple one-component liquids [11] have shown the marked dependence of the dynamic structure factor and propagating longitudinal modes on the softness of the potential core. This investigation has been extended to the case of the binary liquids considered in the present study, and the results will also be discussed in this paper.

\section{DESCRIPTION OF THE MD SIMULATIONS}

MD simulations of different liquid binary mixtures at $T$ $=120 \mathrm{~K}$ were carried out. Six liquid mixtures made up of $N_{1}=648$ particles of species 1 and $N_{2}=216$ particles of species 2 within a cubic box and the usual periodic boundary conditions were considered. The mass of the former (light particles) was $m_{1}=25.95 \mathrm{amu}$, whereas the mass of the second (heavy particles) was $m_{2}=20 m_{1}$. Thus, the mean mass $\left[\mu^{-1 / 2} \equiv x_{1} m_{1}^{-1 / 2}+x_{2} m_{2}^{-1 / 2}[12]\right.$, where $x_{1}=N_{1} /\left(N_{1}+N_{2}\right)$ and $x_{2}=N_{2} /\left(N_{1}+N_{2}\right)$ are the fractional concentration of species 1 and 2 , respectively] of the system was $\mu$ $=39.95$ uma. The densities in the different simulations (Table I) were chosen so that the pressure was close to a fixed value $(p \approx 800 \mathrm{MPa})$. The most important differences among the simulated systems were the interaction potentials $\left[V_{i j}(r) ; i=1,2 ; j=1,2\right]$. The characteristics and parameters of the potentials are summarized in Table I and the $V_{i j}(r)$ functions are depicted in Fig. 2. We started with a system $(A)$, which structure shows analogous features to that of
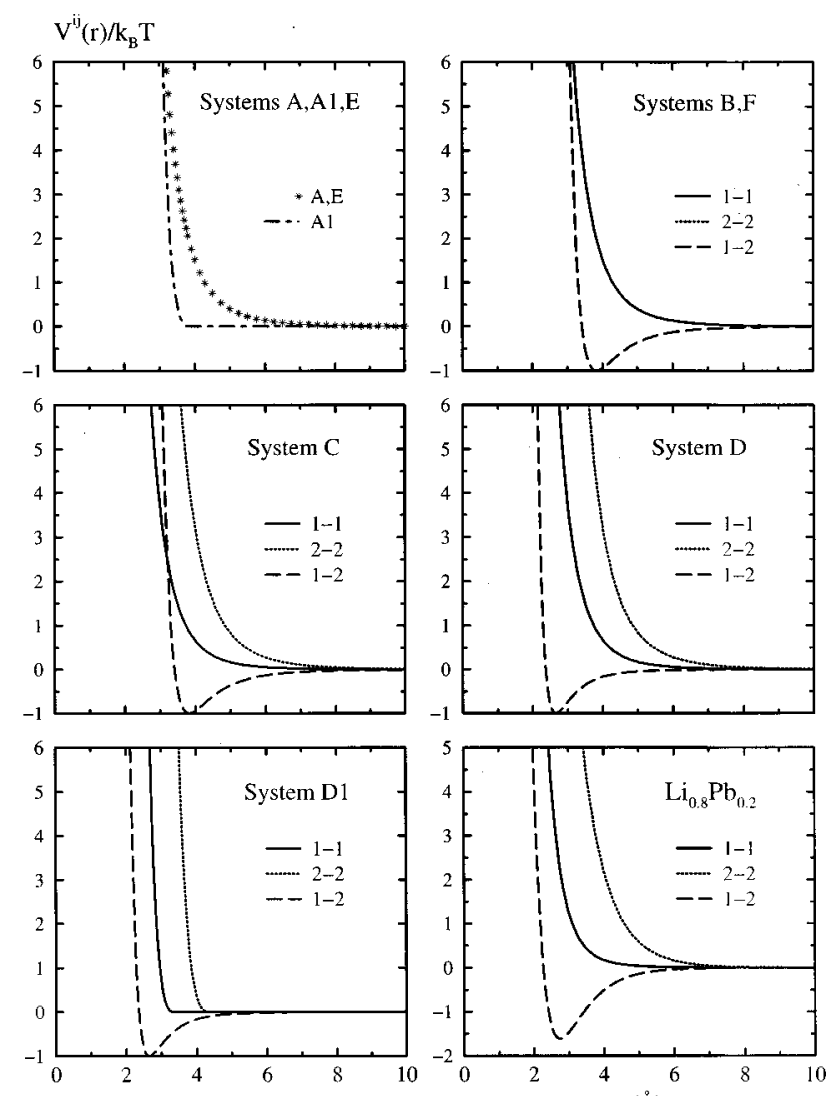

FIG. 2. Interaction potentials used in the MD simulations. Potential $A$ corresponds to a simple alloy model with two species of the same size. Potential $D$ reproduces the most significant structural features of ionic alloys such as $\mathrm{Li}_{0.8} \mathrm{~Pb}_{0.2}$ [7]. Potentials $B$ and $C$ are intermediate between $A$ and $D$. Potentials $A 1$ and $D 1$ are analogous to potentials $A$ and $D$, respectively, but with harder potential cores between like particles. Potentials $E$ and $F$ are identical to potentials $A$ and $B$, respectively. For the potentials $A$ and $A 1: V^{11}(r)$ $\equiv V^{12}(r) \equiv V^{22}(r)$. 
simple binary mixtures of realistic systems, such as the $\mathrm{Li}_{0.7} \mathrm{Mg}_{0.3}$ alloy. The reason for the use of simple potential models instead of more realistic ones, such as the NPA potentials, is that the former are expressed by a simple mathematical function with few parameters that can be suitably modified. Thus, we can pass in successive steps from system $A$ to system $D$, which shows a structure analogous to that of partially ionic alloys such as $\mathrm{Li}_{4} \mathrm{~Pb}$.

In the system $A$, all the particles interact through a repulsive soft-sphere (SS) potential, i.e., $V_{11}(r)=V_{12}(r)$ $=V_{22}(r)=4 \varepsilon(\sigma / r)^{6}$, with $\varepsilon=119.8 \mathrm{~K}$ and $\sigma=3.405 \AA$. This potential is a simple model of a liquid alloy with two species of particles of the same size, i.e., the only difference between species 1 and 2 is the mass of the particles. The cutoff for the interactions was at $2.5 \sigma$. In order to study the influence of the softness of the potential core, another system with identical conditions but a potential with a harder wall was also simulated. This system will be termed $A 1$. In this case, the interactions were the repulsive part of a LennardJones (RLJ) potential $\left(V_{11}(r)=V_{12}(r)=V_{22}(r)\right.$ $\left.=4 \varepsilon\left[(\sigma / r)^{12}-(\sigma / r)^{6}\right]\right)$ truncated at its minimum $r_{\text {min }}$ $=2^{1 / 6} \sigma$. The parameters $\sigma$ and $\varepsilon$ were the same as for the system $A$.

As may be observed in Fig. 2, there are marked differences between the potential ordinarily used for $\mathrm{Li}_{4} \mathrm{~Pb}[7]$ and that used for system $A$. The interaction between $\mathrm{Li}$ and $\mathrm{Pb}$ is attractive at intermediate distances, the effective size of the $\mathrm{Li}$ and $\mathrm{Pb}$ particles is very different, and the interactions between distinct species show large deviations from additivity, i.e., the repulsive wall for the $\mathrm{Li}-\mathrm{Pb}$ interactions is noticeably shifted to the left in relation to the repulsive walls for the $\mathrm{Li}-\mathrm{Li}$ and $\mathrm{Pb}-\mathrm{Pb}$ interactions. These characteristics of the $V_{\mathrm{LiPb}}(r)$ potential reflect the ionic character of the $\mathrm{Li}_{4} \mathrm{~Pb}$ alloy. We modified the potential for system $A$ in three successive steps in order to reproduce separately the most significant features of the potentials for ionic alloys.

Attractive forces between particles 1 and 2 were assumed in system $B$. In this case, both $V_{11}(r)$ and $V_{22}(r)$ were the same as for system $A$ while $V_{12}(r)$ was a Lennard-Jones (LJ) potential truncated at $2.5 \sigma$. The potential parameters $\varepsilon$ and $\sigma$ were the same as for $A$. The effective sizes of particles 1 and 2 were different in system $C$. The $V_{i j}(r)$ functions for this system were the same as for $B$ but $\sigma_{12}=\left(\sigma_{11}+\sigma_{22}\right) / 2$ $=3.405 \AA$ and $\sigma_{22}=1.3 \sigma_{11}$. Finally, the effective sizes of particles were nonadditive in system $D$, i.e., $\sigma_{12}<\left(\sigma_{11}\right.$ $\left.+\sigma_{22}\right) / 2$. It should be noted that the nonadditivity of the sizes of particles is a characteristic of the interactions in systems of charged particles. For system $D$, the $V_{i j}(r)$ functions as well as the parameters $\sigma_{11}$ and $\sigma_{22}$ were the same as for $C$ but $\sigma_{12}=0.8 \sigma_{11}$. As shown in Fig. 2, the main features of the $V_{i j}(r)$ potential functions for $D$ are very similar to those for $\mathrm{Li}_{0.8} \mathrm{~Pb}_{0.2}$, whereas the $V_{i j}(r)$ potential functions for $B$ and $C$ are intermediate. We also performed a simulation of a system identical to system $D$ but with harder potential cores between like particles. This system will be termed $D 1$. As with $A 1$, both $V_{11}(r)$ and $V_{22}(r)$ for $D 1$ were the repulsive part of a Lennard-Jones potential. The potential parameters for $D 1$ were the same parameters as for $D$.

To analyze the dependence of the results on the proportion of heavy and light particles, we simulated two additional mixtures, which will be termed systems $E$ and $F$. These systems were identical to the systems $A$ and $B$, respectively, but with an inverse proportion of light and heavy particles, i.e., they were made up of 216 particles of species 1 and 648 particles of species 2 .

Both the partial radial distribution functions $\left[g^{i j}(r)\right]$ and the $F^{i j}(k, t)$ functions were calculated according to the usual definitions [13] during the MD simulations. The $k$-dependent functions were determined for nine different wave vectors ranging from the lowest value compatible with the size of the box $k \approx 0.3-2.5 \AA^{-1}$. The structure factors $\left[S^{i j}(k)\right]$ and the dynamic structure factors $\left[S^{i j}(k, \omega)\right]$ were obtained by Fourier-transforming $g^{i j}(r)$ and $F^{i j}(k, t)$, respectively. The number density-concentration intermediate scattering functions were determined according to the definition [13]

$$
\begin{gathered}
F^{\mathrm{NN}}(k, t) \equiv x_{1} F^{11}(k, t)+x_{2} F^{22}(k, t)+2\left(x_{1} x_{2}\right)^{1 / 2} F^{12}(k, t) \\
F^{\mathrm{NC}}(k, t) \equiv x_{1} x_{2}\left[F^{11}(k, t)-F^{22}(k, t)+\left(x_{2}-x_{1}\right)\right. \\
\left.\times\left(x_{1} x_{2}\right)^{-1 / 2} F^{12}(k, t)\right] \\
F^{\mathrm{CC}}(k, t) \equiv x_{1} x_{2}\left[x_{2} F^{11}(k, t)+x_{1} F^{22}(k, t)\right. \\
\left.-2\left(x_{1} x_{2}\right)^{1 / 2} F^{12}(k, t)\right]
\end{gathered}
$$

The $F^{\mathrm{NN}}(k, t)$ functions describe the averaged behavior of the system, and in the limit case of a mixture of two identical species they should be identical to the $F(k, t)$ functions for the corresponding one-component system. As with onecomponent liquids, $S^{\mathrm{NN}}(k, \omega)$ at low $k$ show a Rayleigh peak centered at $\omega=0$ and two shifted Brillouin peaks at $\omega$ $= \pm c_{s} k$. The Brillouin peaks correspond to propagating sound modes with velocity $c_{s}$. While the $F^{\mathrm{NN}}(k, t)$ functions are related to the number-density fluctuations, the $F^{\mathrm{CC}}(k, t)$ correlations are associated with the fluctuations caused by the opposite motions of particles of different species. The concentration-concentration correlations are analogous to the charge-charge correlations in ionic solids and liquids. The peaks in the spectra of the charge-charge correlation functions for ionic systems [14] are associated with propagating optic modes of kinetic character, i.e., the frequency goes to a finite value as $k$ goes to zero.

\section{STRUCTURE}

The resulting $g^{i j}(r)$ functions are depicted in Fig. 3. The three partial $g^{i j}(r)$ 's for system $A$ are identical since the only difference between the two species is the atomic mass. Moreover, they show the typical features of the $g(r)$ in dense simple liquids. The $g^{i j}(r)$ 's for systems $A$ and $A 1$ only show slight differences in the height of the first peak, which indicates that the influence of the hardness of the potential core on the structure is rather weak. However, the attractive interactions between unlike particles produce marked changes in the structure. The first maximum of the $g^{22}(r)$ function for both system $B$ and system $C$ becomes substantially shifted towards higher $r$ values and corresponds to configurations with a light particle between two heavy particles. The little 

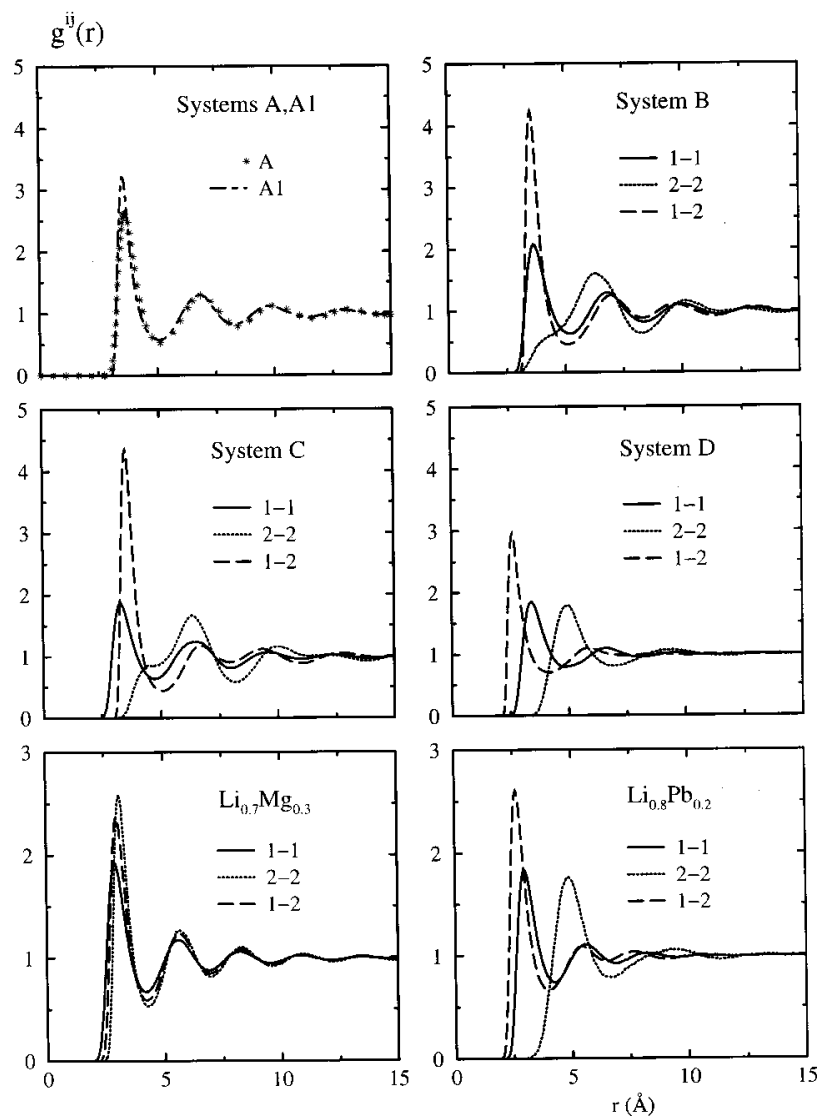

FIG. 3. Partial radial distribution functions for different systems. For the systems $A$ and $A 1 ; g^{11}(r) \equiv g^{12}(r) \equiv g^{22}(r)$.

shoulder in $g^{22}(r)$ at distances close to the $V_{22}(r)$ wall reflects that two heavy particles are rarely in contact. On the contrary, $g^{11}(r)$ has a clear first maximum at short distances, which shows that configurations with two close light particles are frequent. These findings are consistent with the higher proportion of light particles in the mixture. The structure of system $D$ is markedly different from that of system $C$. As can be expected, the maximum of $g^{12}(r)$ for system $D$ is shifted towards lower $r$ values while the changes for the $g^{22}(r)$ function are more significant. $g^{22}(r)$ for system $D$ shows a first peak associated with contact configurations. Moreover, the position of this maximum is close to that of the first minimum of $g^{11}(r)$ while the first maximum of $g^{11}(r)$ is close to the first minimum of $g^{12}(r)$. This indicates that the structure of system $D$ presents some incipient features of the $A B A B$ ordering as found in molten salts. Because of the very different proportion of the two species in the mixture and the large size differences, the $g^{i j}(r)$ functions for system $D$ show marked discrepancies with those for simple molten salts such as $\mathrm{NaCl}$ [15] but similar features with the $g^{i j}(r)$ 's for less symmetric molten salts such as $\mathrm{SrCl}_{2}[16]$.

The partial structure factors $S^{i j}(k)$ were calculated by Fourier-transforming the $g^{i j}(r)$ functions that were directly obtained from the MD simulations. As may be observed from Fig. 4, the $S^{i j}(k)$ functions allow us to see more clearly the significant changes that the attractive interactions be-

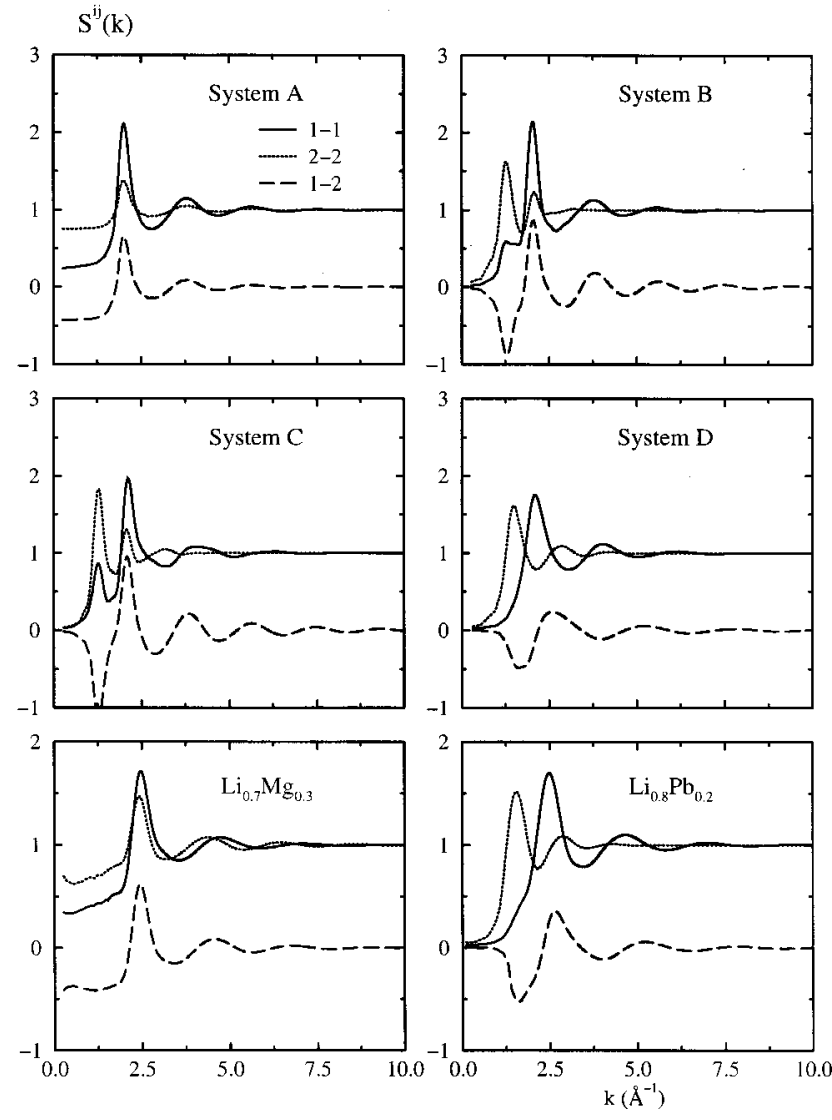

FIG. 4. Partial static structure factors for different systems.

tween distinct particles produce on the structure of the binary mixtures. The analysis of the $S^{i j}(k)$ functions at low $k$ 's is interesting because the initial values of the $F^{i j}(k, t)$ functions should coincide with $S^{i j}(k)$. For both system $A$ and the $\mathrm{Li}_{0.7} \mathrm{Mg}_{0.3}$ alloy, the three $S^{i j}(k)$ functions show different and relatively high values as $k$ goes to zero $\left[S^{11}(0)\right.$ $\left.>0 ; S^{22}(0)>0 ; S^{12}(0)<0\right]$. On the contrary, for systems $B$, $C, D$, and $\mathrm{Li}_{0.8} \mathrm{~Pb}_{0.2}$, the three $S^{i j}(k)$ functions are very close and have very small values as $k$ goes to zero $\left[\left|S^{11}(0)\right|\right.$ $\left.\approx\left|S^{22}(0)\right| \approx\left|S^{12}(0)\right| \ll 1\right]$. The large differences between the values of the $F^{i j}(k, t)$ functions for $\mathrm{Li}_{0.8} \mathrm{~Pb}_{0.2}$ and $\mathrm{Li}_{0.7} \mathrm{Mg}_{0.3}$ may then be related to a greater coherence between distinct particles in $\mathrm{Li}_{0.8} \mathrm{~Pb}_{0.2}$ due to the ionic character of this mixture. It should be emphasized that in the ioniclike systems $B$, $C$, and $D$ as well as in $\mathrm{Li}_{0.8} \mathrm{~Pb}_{0.2}$, there is a notable asymmetry in the interactions between like and unlike particles, the former being purely repulsive and the latter being attractive at intermediate distances (see Fig. 2). The ioniclike character of systems $B, C$, and $D$ is also revealed by the $S^{12}(k)$ functions that show a deep first minimum analogous to that typical of the molten salts $[15,17]$. On the contrary, $S^{12}(k)$ for system $A$ only shows the characteristic peak of the random liquid mixtures [17].

The number density-concentration factors $S^{\mathrm{NN}}(k)$, $S^{\mathrm{NC}}(k)$, and $S^{\mathrm{CC}}(k)$ shown in Fig. 5 were calculated as a combination of the partial $S^{i j}(k)$ factors according to Eq. (1). $S^{\mathrm{NN}}(k)$ for system $A$ shows the ordinary shape of the structure factor in dense one-component fluids. A similar behavior 

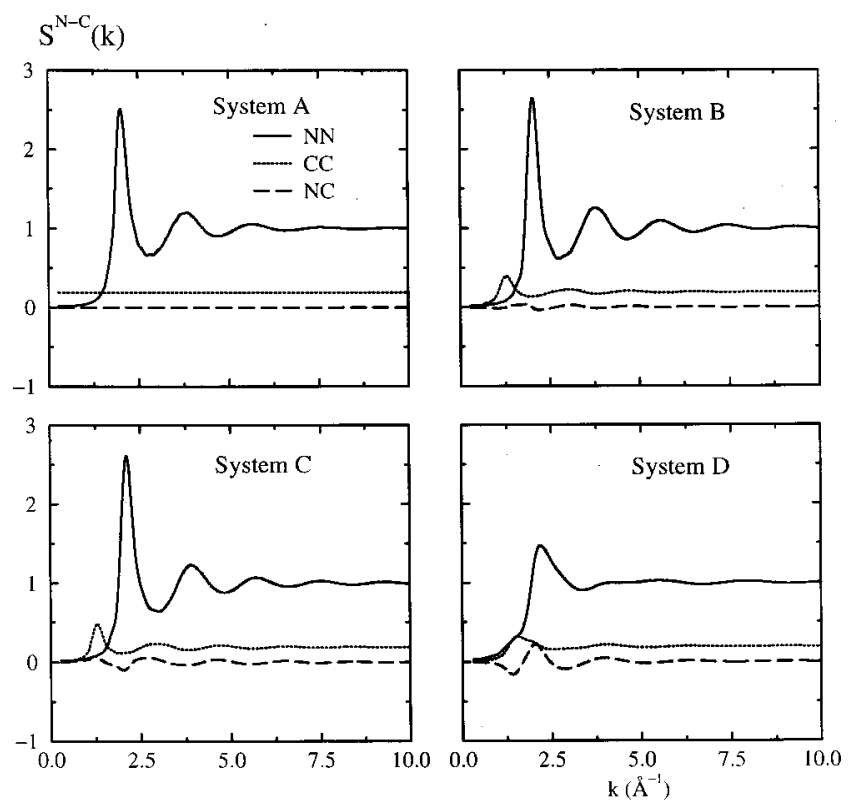

FIG. 5. Number density-concentration static structure factors for different systems.

is shown by the $S^{\mathrm{NN}}(k)^{\prime}$ 's for systems $B$ and $C$, whereas the first peak and subsequent oscillations of $S^{\mathrm{NN}}(k)$ are much less marked for system $D$. The shape of the $S^{\mathrm{NN}}(k)$ factor for system $D$ reflects the existence of a rudimentary layer structure $(A B A B$ ordering) in this system. It should be noted that $S^{\mathrm{NN}}(k)$ for simple molten salts is rather structureless $[15,17]$, whereas for other ionic materials such as $\mathrm{SrCl}_{2}, \mathrm{ZnCl}_{2}$, and $\mathrm{Ag}_{2} \mathrm{Se}$ it is even more structured than that for system $D$ [18]. We want to emphasize the lack of structure associated with concentration fluctuations in system $A$, which reveals that the relative positions of particles of the two species in this mixture are randomly distributed. On the contrary, the $S^{\mathrm{CC}}(k)$ factors for systems $B, C$, and $D$ show a noticeable peak that, however, is much lower than the strong $S^{\mathrm{CC}}(k)$ peak characteristic of ionic liquids with a rather long-range-ordered structure $[15,17]$. The number density-concentration correlations are very small for systems $B$ and $C$ but somewhat higher in the case of the "more ordered" system $D$.

\section{DYNAMICS}

\section{A. Intermediate scattering functions}

The partial $F^{i j}(k, t)$ functions at the lowest wave vectors considered in this study are plotted in Fig. 6. As with $\mathrm{Li}_{0.7} \mathrm{Mg}_{0.3}$ (Fig. 1), the $F^{i j}(k, t)$ functions for system $A$ show large initial values and decay very slowly without noticeable oscillations. Nevertheless, the inclusion of attractive forces between unlike particles produces dramatic changes in the $F^{i j}(k, t)$ functions. The initial values of the $F^{i j}(k, t)$ 's for systems $B$ and $C$ (Fig. 6) are one order of magnitude lower than those for system $A$, which is consistent with the small values of the $S^{i j}(k)$ functions for these systems at low wave vectors (Fig. 4). However, the decay of the $F^{i j}(k, t)$ 's for systems $B$ and $C$ is rather slow, whereas the $F^{i j}(k, t)$ 's for

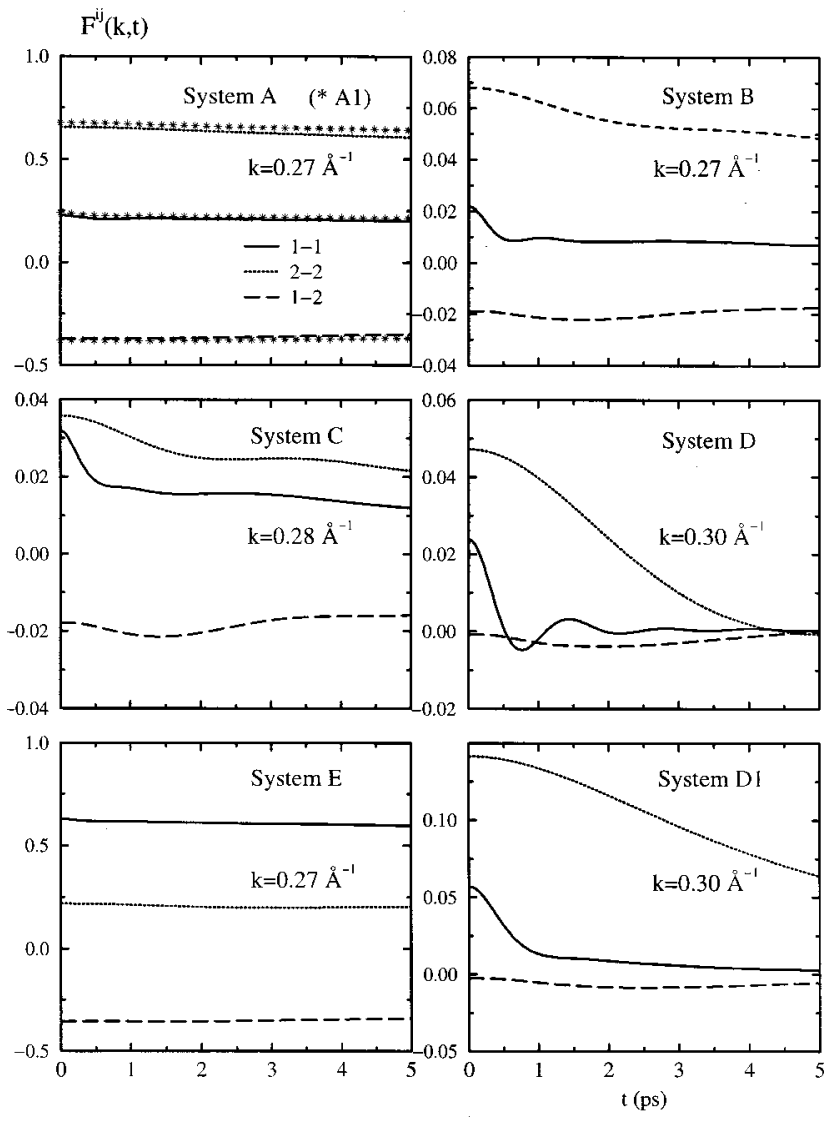

FIG. 6. Partial intermediate scattering functions for different systems at the lowest wave vector in this study.

system $D$ show faster decays and their spectra can be easily calculated. The comparison of Figs. 1 and 6 corroborates that the $F^{i j}(k, t)$ functions for both system $D$ and $\mathrm{Li}_{0.8} \mathrm{~Pb}_{0.2}$ show very close trends. It should be emphasized that the values of the $F^{\mathrm{NN}}(k, t)$ functions for systems $A$ and $D$, which represent the overall behavior of the mixtures, are of the same order of magnitude and their shapes are similar (Fig. 8). This corroborates recent findings that suggested that the large initial values as well as the slow decays of the three $F^{i j}(k, t)$ functions are largely canceled when the $F^{\mathrm{NN}}(k, t)$ functions are computed [6].

In order to discuss the origin of the dramatic differences between the $F^{i j}(k, t)$ functions for systems $A$ and $D$ at low $k$ 's, it is interesting to separate the total $F^{i j}(k, t)$ functions into the self and distinct components $[13,15]$.

$$
\begin{gathered}
F^{i i}(k, t)=F_{s}^{i}(k, t)+F_{d}^{i i}(k, t) \quad(i=1,2), \\
F^{i j}(k, t)=F_{d}^{i j}(k, t) \quad(i \neq j) .
\end{gathered}
$$

As shown in Fig. 7, in the case of system $D$ both $F_{d}^{11}(k, t)$ and $F_{d}^{22}(k, t)$ are almost identical but with opposite sign to $F_{s}^{1}(k, t)$ and $F_{s}^{2}(k, t)$, respectively. These features are also characteristic of the self and distinct intermediate scattering functions in one-component liquids. Besides, $F_{d}^{12}(k, t)$ is much smaller than $F_{d}^{11}(k, t)$ and $F_{d}^{22}(k, t)$. Similar findings were obtained for the $\mathrm{Li}_{0.8} \mathrm{~Pb}_{0.2}$ alloy as well as for molten 

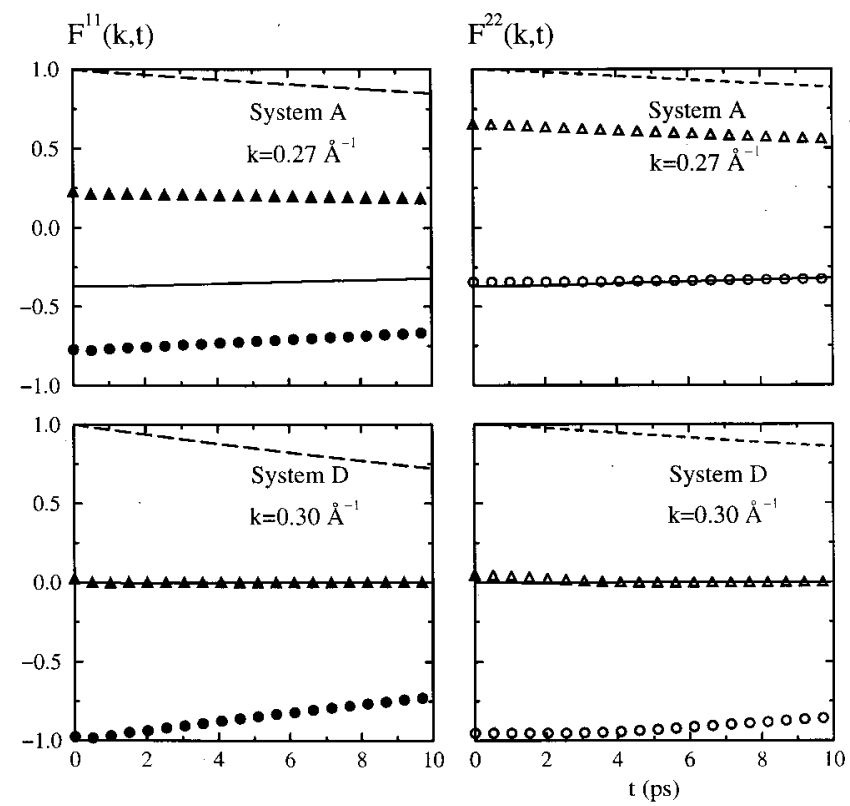

FIG. 7. Self and distinct components of the $F^{i j}(k, t)$ partial intermediate scattering functions for systems $A$ and $D$. Full and open triangles, $F^{11}(k, t)$ and $F^{22}(k, t)$. Full and open circles, $F_{d}^{11}(k, t)$ and $F_{d}^{22}(k, t)$. Continuous line, $F_{d}^{12}(k, t)$. Long dashed and dashed lines, $F_{s}^{1}(k, t)$ and $F_{s}^{2}(k, t)$.

salts [19]. These results suggest that the density fluctuations at low $k$ 's corresponding to the subset of particles of one the species in binary liquids with $A B A B$ ordering are analogous to those in one-component liquids. On the contrary, the density fluctuations in binary liquids where particles of the two species are randomly distributed are similar to those for a dilute liquid or a gas, as shown by the results for system $A$ in Fig. 7 (analogous results were obtained for the $\mathrm{Li}_{0.7} \mathrm{Mg}_{0.3}$ alloy). In these liquid mixtures, the components of the total intermediate functions for particles of one of the species present important differences in relation to those in pure liquids, i.e., $F_{d}^{11}(k, t), F_{d}^{12}(k, t)$, and $F_{d}^{22}(k, t)$ have similar values, which in turn are markedly different from the $F_{s}^{1}(k, t)$ and $F_{s}^{2}(k, t)$ values.

The $F^{i j}(k, t)$ functions do not show significant changes with the softness of the potential cores. The results for $A 1$ are very close to those for $A$, whereas the $F^{i j}(k, t)$ 's for $D 1$ show slightly greater values and slower decays than for $D$. The large cancellations between the partial $F^{i j}(k, t)$ when $F^{\mathrm{NN}}(k, t)$ is calculated may also be observed for system $A 1$ (Fig. 8).

\section{B. Diffusion coefficients}

The values of self-diffusion coefficients $D_{S}^{1}$ and $D_{S}^{2}$ obtained from the mean-square displacements of the light and heavy particles, respectively, are summarized in Table II. The interdiffusion coefficients $D_{I}^{12}$ have been estimated according to the approximate relation [15]

$$
D_{I}^{12}=x_{1} x_{2}\left(x_{1} D_{S}^{2}+x_{2} D_{S}^{1}\right) / S^{\mathrm{CC}}(0)
$$

and their values are also given in Table II.

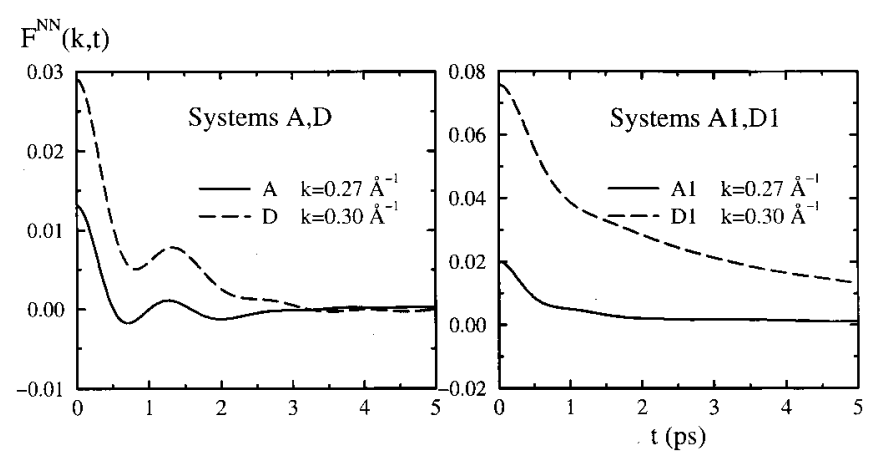

FIG. 8. Comparison of the number density-number density intermediate scattering functions for systems $A, A 1, D$, and $D 1$.

It should be emphasized that the interdiffusion coefficients for systems $B, C$, and $D$ are markedly higher than for system $A$, especially in the case of system $D$. This suggests that particles can spread more easily in the more ordered system $D$, which is consistent with the faster decay of the $F^{i j}(k, t)$ functions for this system. The results in Table II prove that the diffusion coefficients are lower when the heavy particles are in the majority than when they are in the minority. We have not observed any clear dependence of the diffusion coefficients on the hardness of the potential cores.

\section{Dynamic structure factors}

Results in Figs. 6 and 8 suggest that the oscillations of the $F^{i j}(k, t)$ 's for system $D$ [especially those of $\left.F^{11}(k, t)\right]$ as well as the corresponding $S^{i j}(k, \omega)$ maxima could also be present for the other systems, including system $A$. To check this point, we used the same procedure as in Ref. [6]. It is assumed that the long time decay of $F^{i j}(k, t)$ may be approximately represented by an exponential function. Thus, it is defined as

$$
F_{-}^{i j}(k, t)=F^{i j}(k, t)-A_{i j} \exp \left(-\alpha_{i j} t\right),
$$

where $A_{i j}$ and $\alpha_{i j}$ are $k$-dependent constants that were determined by fitting $A_{i j} \exp \left(-\alpha_{i j} t\right)$ to the long time part of $F^{i j}(k, t)$. The $F_{-}^{i j}(k, t)$ function keeps both the oscillatory and the fast-decaying components of $F^{i j}(k, t)$, whereas the slow-decaying component is mostly removed. $S_{-}^{i j}(k, \omega)$ is then obtained as the Fourier transform of $F_{-}^{i j}(k, t)$. As may be seen in Fig. 9, the $S_{-}^{11}(k, \omega)$ functions for system $A$ show a noticeable peak for frequencies very close to those of the $S^{11}(k, \omega)$ peak for the same wave vector. This proves that the peaks in the $S^{\mathrm{LiLi}}(k, \omega)$ for $\mathrm{Li}_{0.8} \mathrm{~Pb}_{0.2}$, which were associated with fast-propagating modes, are also present in other liquid mixtures, although in many systems they cannot be easily observed because they remain hidden due to other large and slowly decaying contributions to the $F^{i j}(k, t)$ functions that are also dominant in the $S^{i j}(k, \omega)$ spectra.

As shown in Fig. 10, the $S^{11}(k, \omega)$ functions for system $D$ present a clear Brillouin peak up to rather high wave vectors. The frequencies corresponding to these peaks are also high and their values increase with $k$. These results, which are very similar to those for $\mathrm{Li}_{0.8} \mathrm{~Pb}_{0.2}[1]$, reflect the existence of 
TABLE II. Diffusion coefficients in $10^{-9} \mathrm{~m}^{2} \mathrm{~g}^{-1}$.

\begin{tabular}{|c|c|c|c|c|c|c|c|c|}
\hline & $\begin{array}{c}\text { System } \\
A\end{array}$ & $\begin{array}{c}\text { System } \\
A 1\end{array}$ & $\begin{array}{c}\text { System } \\
B\end{array}$ & $\begin{array}{c}\text { System } \\
C\end{array}$ & $\begin{array}{c}\text { System } \\
D\end{array}$ & $\begin{array}{c}\text { System } \\
D 1\end{array}$ & $\begin{array}{c}\text { System } \\
E\end{array}$ & $\begin{array}{c}\text { System } \\
F\end{array}$ \\
\hline$D_{S}^{1}$ & 2.2 & 1.4 & 1.1 & 1.0 & 3.7 & 4.1 & 1.1 & 0.4 \\
\hline$D_{S}^{2}$ & 1.8 & 1.1 & 0.6 & 0.5 & 1.9 & 2.4 & 0.9 & 0.5 \\
\hline$D_{I}^{12}$ & 1.9 & 1.2 & 10.5 & 13.5 & 57.6 & 23.5 & 1.0 & 5.3 \\
\hline
\end{tabular}

fast-propagating modes through light particles. In the case of system $D 1$, the $F^{11}(k, t)$ 's have a fast decay and the corresponding spectra could be determined. However, they do not show any noticeable peak within the $k$ region considered in this study (Fig. 10), which is consistent with the nonoscillatory $F^{\mathrm{NN}}(k, t)$ function obtained for the lowest $k$ value (Fig. $8)$. This result indicates that lower wave vectors should be analyzed to observe the Brillouin peaks in system $D 1$ [as with one-component liquids, a Brillouin peak should be observed in $S^{\mathrm{NN}}(k, \omega)$ at the hydrodynamic limit]. The $S^{11}(k, \omega)$ results for systems $D$ and $D 1$ in Fig. 10 are then consistent with the findings for one-component liquids [11], which proved that longitudinal modes propagate up to higher wave vectors in liquids with softer potential cores.
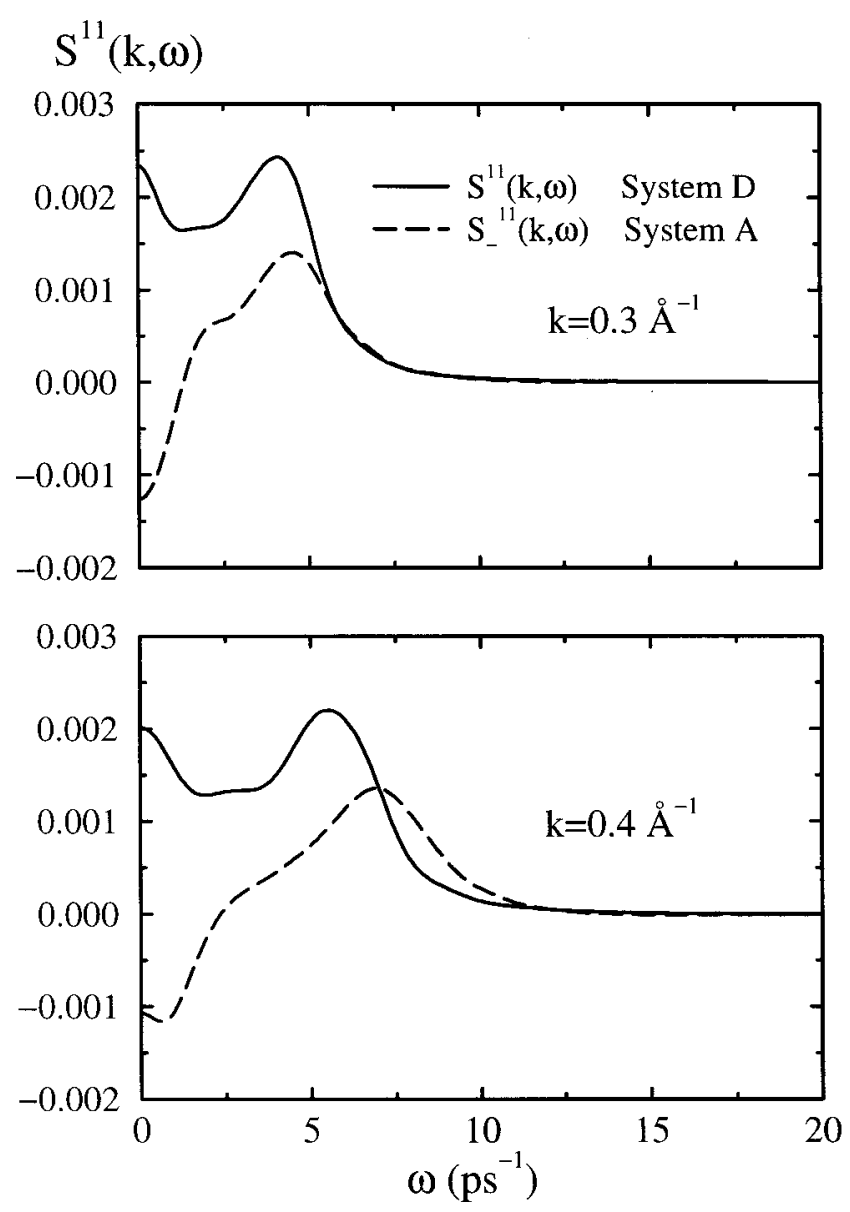

FIG. 9. Dynamic structure factors for the light particles $\left[S^{11}(k, \omega)\right]$ in system $D$ compared with the corresponding $S^{11}(k, \omega)$ functions (see the definition in the text) in system $A$.

\section{Longitudinal dispersion relations}

The longitudinal current correlation functions $C_{L}(k, t)$ and corresponding spectra $C_{L}(k, \omega)$, which were calculated according to the usual definitions [13], are very useful to analyze the longitudinal modes in liquids. Although there is a simple relation between them, $C_{L}(k, \omega)=\omega^{2} S(k, \omega)$, the low-frequency part of $S(k, \omega)$ is absent in $C_{L}(k, \omega)$ whereas the high-frequency part of the former becomes enhanced in the second. Besides, $C_{L}(k, \omega)$ has a visible peak at any $k$ that at low $k$ 's has the same or slightly higher frequency than the Brillouin peak in $S(k, \omega)$. Thus, the longitudinal dispersion relations $\omega_{L}(k)$ may be quite easily obtained from the positions of the $C_{L}(k, \omega)$ maxima for the different wave vectors.
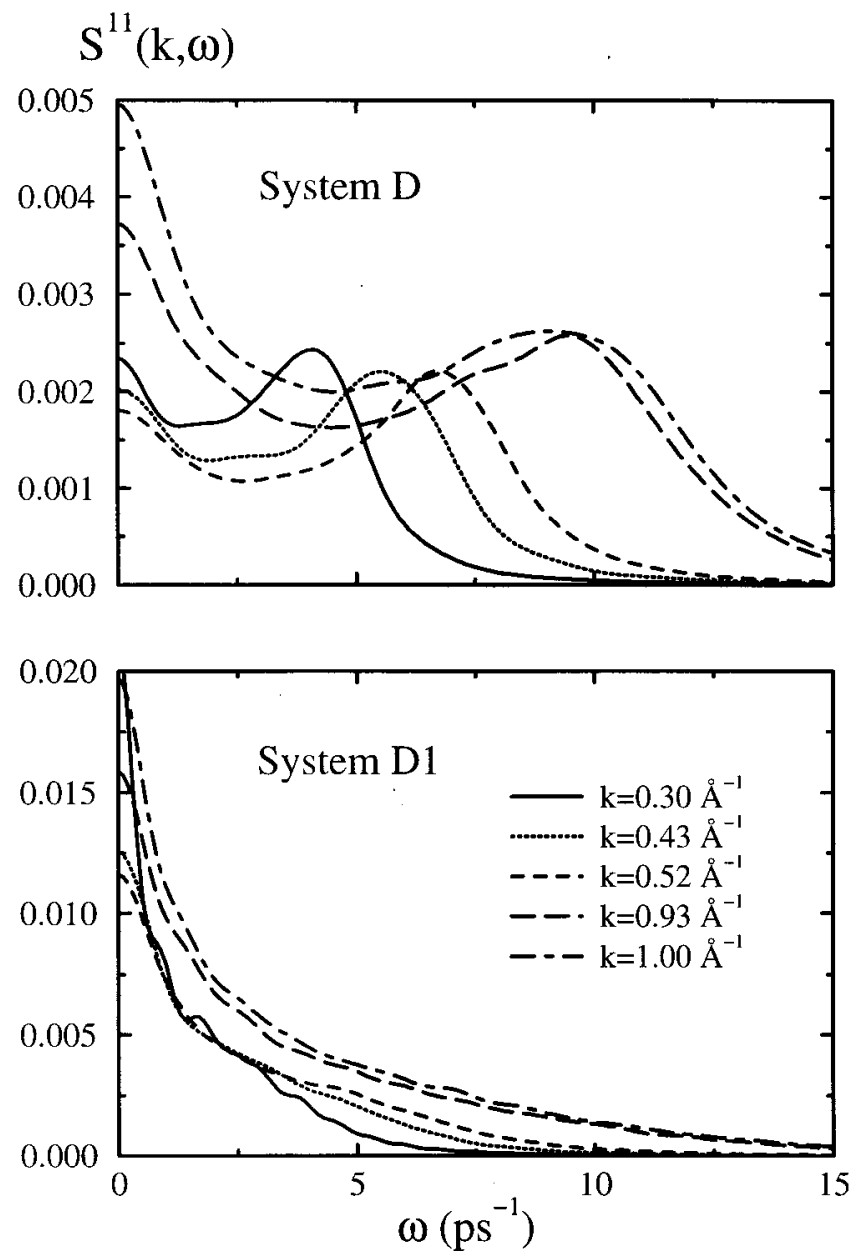

FIG. 10. Dynamic structure factors for light particles at different wave vectors for systems $D$ and $D 1$. 

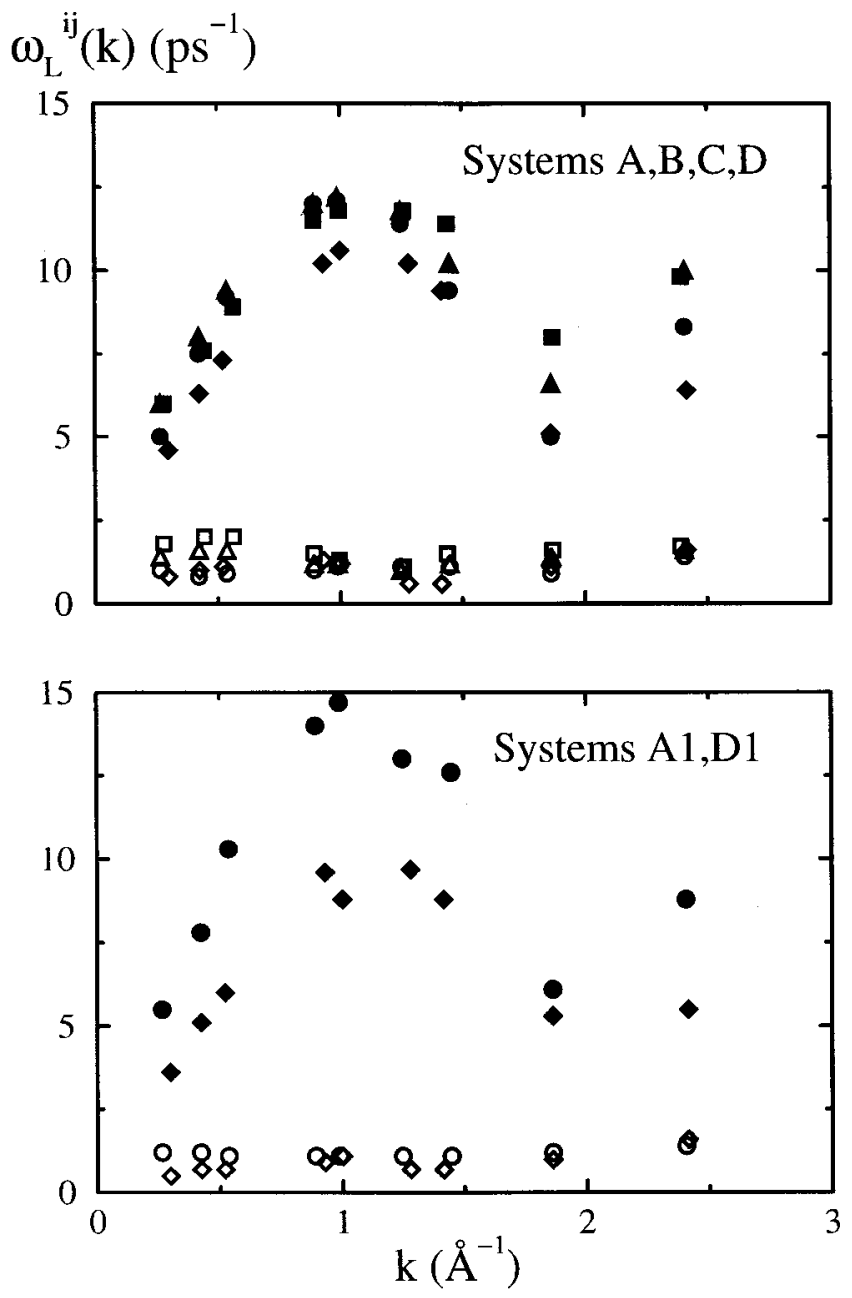

FIG. 11. Dispersion relations corresponding to the longitudinal partial currents. Full symbols, $\omega_{L}^{11}(k)$; open symbols, $\omega_{L}^{22}(k)$; circles, systems $A, A 1$; triangles, system $B$; squares, system $C$; diamonds, system $D$.

The partial $C_{L}^{i j}(k, t)$ functions and corresponding spectra $C_{L}^{i j}(k, \omega)$ were obtained for the different binary liquids in this study and the resulting relation dispersions $\omega_{L}^{i j}(k)$ are illustrated in Figs. 11 and 12.

The $\omega_{L}^{11}(k)$ and $\omega_{L}^{22}(k)$ functions depicted in Fig. 11 show that there are no large deviations between the results for the different systems. These findings reflect that, unlike other properties such as the structure or the intermediate scattering functions, the relation dispersions are rather insensitive to the details of the interatomic potentials. As with one-component liquids [9], the values of $\omega_{L}^{i j}(k)$ should be mainly associated with the thermodynamic state of the system and the atomic mass of particles. The large discrepancies between $\omega_{L}^{11}(k)$ and $\omega_{L}^{22}(k)$ should then be attributed to the mass difference between the particles of the two species. The values of $\omega_{L}^{11}(k)$ and $\omega_{L}^{22}(k)$ are very different at all $k$ 's, which shows that the lowest wave vectors considered in this study are still far from the hydrodynamic region where the frequencies for the two species should coincide $[5,6]$. This is consistent with earlier findings that suggested that the $k$ value required to

$$
\omega_{\mathrm{L}}(\mathrm{k})\left(\mathrm{ps}^{-1}\right)
$$
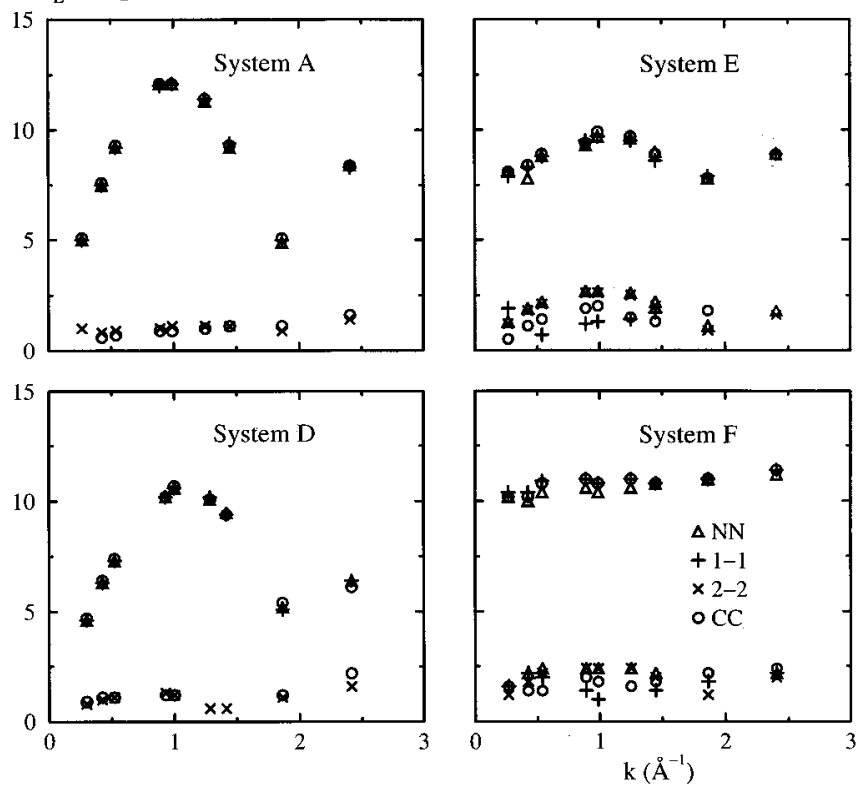

FIG. 12. Longitudinal dispersion relations corresponding to the partial currents $\left[\omega_{L}^{11}(k), \omega_{L}^{22}(k)\right]$ and the number densityconcentration currents $\left[\omega_{L}^{\mathrm{NN}}(k), \omega_{L}^{\mathrm{CC}}(k)\right]$.

reach the hydrodynamic regime decreases when the mass ratio of the species in the mixture increases [6].

The values of $\omega_{L}^{22}(k)$ for systems $E$ and $F$ (Fig. 12), which now correspond to the majority particles, are very close to those for the other systems. The $C_{L}^{11}(k, \omega)$ functions for systems $E$ and $F$ present two peaks. The low $\omega_{L}^{11}(k)$ value coincides with the $\omega_{L}^{22}(k)$ value for the same $k$, whereas the high $\omega_{L}^{11}(k)$ value is of the same order of magnitude as $\omega_{L}^{11}(k)$ for the systems in which light particles are in the majority but remains almost independent of $k$ (Fig. 12). These findings suggest that in liquid mixtures of disparate-mass particles at different compositions, the heavy particles always keep their characteristic low frequencies whereas light particles keep their high frequencies but also catch the low frequencies of the heavy particles when they are in the minority.

The $\omega_{L}^{\mathrm{NN}}(k)$ dispersion curves (Fig. 12) obtained from the peaks of the $C_{L}^{\mathrm{NN}}(k, \omega)$ functions are generally very close to the dispersion curves corresponding to the majority particles. However, in the case of systems $E$ and $F$, the $C_{L}^{\mathrm{NN}}(k, \omega)$ functions show a second peak at higher frequency and then two $\omega_{L}^{\mathrm{NN}}(k)$ dispersion curves are obtained, one close to $\omega_{L}^{22}(k)$ and the other close to $\omega_{L}^{11}(k)$. It is also interesting to investigate the existence of concentration modes analogous to the charge modes (optic modes) in molten salts. In general, the $C_{L}^{\mathrm{CC}}(k, \omega)$ functions show two peaks and the corresponding $\omega_{L}^{\mathrm{CC}}(k)$ dispersion curves are very close to $\omega_{L}^{11}(k)$ and $\omega_{L}^{22}(k)$, respectively. According to earlier findings [6], the frequencies of the concentration-concentration modes go to a finite value as $k$ goes to zero, which is a characteristic feature of the kinetic modes that is also shown by optic modes in molten salts [14]. Then, the results in Fig. 12 cor- 
roborate the kinetic character of both the fast- and the slowpropagating modes at intermediate wave vectors that are associated with the $\omega_{L}^{11}(k)$ and $\omega_{L}^{22}(k)$ dispersion curves, respectively. The larger the difference is between the mass of the particles, the bigger is the difference between the $\omega_{L}^{11}(k)$ and $\omega_{L}^{22}(k)$ frequencies. In our mixtures of disparate-mass particles, acousticlike modes with coincident $\omega_{L}^{11}(k)$ and $\omega_{L}^{22}(k)$ values can only be observed at wave vectors much lower than those considered in this study (within the hydrodynamic region).

\section{CONCLUDING REMARKS}

The structure and dynamic properties of two kinds of liquid binary mixtures have been discussed in this paper. On the one hand, we explored simple mixtures with a "random structure," such as the $\mathrm{Li}_{0.7} \mathrm{Mg}_{0.3}$ alloy or system $A$, which were simulated using potential functions that do not show relevant differences for the interactions between like and unlike particles. In these systems, the fluctuations of concentration are negligible and the three partial $S^{i j}(k)$ factors go independently to quite high values as $k$ goes to zero. Then, the values of the partial $F^{i j}(k, t)$ functions are large and the partial $S^{i j}(k, \omega)$ factors can hardly be determined. It should be noted that as a result of large cancellations, the $F^{\mathrm{NN}}(k, t)$ functions for these systems have low values and show noticeable oscillations at small wave vectors. On the other hand, we explored partially ionic mixtures with an "incipient ordered structure" such as the $\mathrm{Li}_{0.8} \mathrm{~Pb}_{0.2}$ alloy or system $D$. In these systems, the potential functions for the interactions between like and unlike particles are different. Forces between particles of different species are attractive at intermediate distances and forces between particles of the same species are purely repulsive. In this case, the properties of the subsystem of particles of the same species show a noticeable similarity with the corresponding properties for onecomponent liquids, i.e., the three partial $S^{i j}(k)$ factors go to very close and small values as $k$ goes to zero, the self and distinct contributions to the $F^{i i}(k, t)$ functions have similar values but of opposite sign, and the $F^{i j}(k, t)$ functions show very low values. Moreover, noticeable concentration fluctuations, which are clearly reflected in the $S^{\mathrm{CC}}(k, \omega)$ functions, were observed for these systems. We can then conclude that the facility for observing fast-propagating modes from the
$S^{11}(k, \omega)$ functions for $\mathrm{Li}_{0.8} \mathrm{~Pb}_{0.2}$ should be mainly attributed to the incipient $A B A B$ ordering in this alloy. However, it should be emphasized that this should not be associated with the existence of long-ranged interactions but to the rather ordered structure at short distances, since fast-propagating modes may also be easily observed in binary mixtures of particles interacting through short-ranged potentials such as system $D$.

It has been shown that the $F^{i j}(k, t)$ 's at low $k$ 's include oscillatory components of similar frequencies for both $\mathrm{Li}_{0.7} \mathrm{Mg}_{0.3}$-like and $\mathrm{Li}_{0.8} \mathrm{~Pb}_{0.2}$-like mixtures at analogous conditions. Nevertheless, in the $\mathrm{Li}_{0.7} \mathrm{Mg}_{0.3}$-like mixtures, the $F^{i j}(k, t)$ oscillations and consequent $S^{i j}(k, \omega)$ peaks remain hidden by low-frequency components. These low-frequency components do not contribute to the longitudinal current spectra and similar longitudinal dispersion relations are obtained for the two types of mixtures. In general, both $C_{L}^{11}(k, \omega)$ and $C_{L}^{22}(k, \omega)$ functions show peaks at intermediate $k$ 's, which reflect the existence of fast- and slowpropagating modes of kinetic character that are associated with light and heavy particles, respectively. Acoustic modes would only appear within the hydrodynamic region, which corresponds to very small $k-\omega$ in the case of disparate-mass liquid mixtures. The frequencies of the longitudinal modes at intermediate $k$ 's depend on both the mass of the particles of distinct species and the thermodynamic state of the system, whereas they are rather independent of the detailed features of the interatomic potentials and the consequent structural characteristics.

A comparison of the results for mixtures with reverse concentrations has shown that light particles may catch the characteristic frequencies of heavy particles when they are in the minority, whereas the opposite does not happen. Finally, it has been proved that the softer the potential cores are, the larger are the wave vectors at which the $S^{i j}(k, \omega)$ functions show noticeable peaks, which is consistent with earlier findings for one-component liquids [11].

\section{ACKNOWLEDGMENTS}

The financial support from the DGICYT of the Spanish government (Grant No. BFM2000-0596-C03-01) and the CIRIT of the "Generalitat de Catalunya" (Grant No. 1999SGR-00146) is gratefully acknowledged.
[1] J. Bosse, G. Jacucci, M. Ronchetti, and W. Schirmacher, Phys. Rev. Lett. 57, 3277 (1986).

[2] P. H. K. de Jong, P. Verkerk, C. F. de Vroege, L. A. de Graaf, W. S. Howells, and S. M. Bennington, J. Phys.: Condens. Matter 6, L681 (1994); M. Alvarez, F. J. Bermejo, P. Verkerk, and B. Roessli, Phys. Rev. Lett. 80, 2141 (1998).

[3] W. Montfrooij, P. Westerhuijs, V. O. de Haan, and I. M. de Schepper, Phys. Rev. Lett. 63, 544 (1989); P. Westerhuijs, W. Montfrooij, L. A. de Graaf, and I. M. de Schepper, Phys. Rev. A 45, 3749 (1992); H. E. Smorenburg, R. M. Crevecoeur, and I. M. de Schepper, Phys. Lett. A 211, 118 (1996); E. Enciso, N.
G. Almarza, P. Dominguez, M. A. González, and F. J. Bermejo, Phys. Rev. Lett. 74, 4233 (1995); T. Bryk, I. Mryglod, and G. Kahl, Phys. Rev. E 56, 2903 (1997).

[4] A. Campa and E. G. D. Cohen, Phys. Rev. A 41, 5451 (1990), and references therein.

[5] R. Fernández-Perea, M. Alvarez, F. J. Bermejo, P. Verkerk, B. Roessli, and E. Enciso, Phys. Rev. E 58, 4568 (1998); E. Enciso, N. G. Almarza, M. A. González, F. J. Bermejo, R. Fernández-Perea, and F. Bresme, Phys. Rev. Lett. 81, 4432 (1998).

[6] N. Anento and J. A. Padró, Phys. Rev. B 62, 11428 (2000). 
[7] A. P. Copestake, R. Evans, H. Ruppersberg, and W. Schirmacher, J. Phys. F: Met. Phys. 13, 1993 (1983).

[8] L. E. González, D. J. González, M. Silbert, and J. A. Alonso, J. Phys.: Condens. Matter 5, 4283 (1993).

[9] M. Canales, D. J. González, L. E. González, and J. A. Padró, Phys. Rev. E 58, 4747 (1998).

[10] G. Jacucci, M. Ronchetti, and W. Schirmacher, J. Phys. (Paris), Colloq. 8, C-385 (1985).

[11] N. Anento, J. A. Padró, and M. Canales, J. Chem. Phys. 111, 10210 (1999).

[12] J. A. Padró, M. Canales, G. Sesé, and A. Giró, Physica A 148, 253 (1988).

[13] N. H. March and M. P. Tosi, Atomic Dynamics in Liquids (MacMillan, London, 1976).
[14] E. M. Adams, I. R. McDonald, and K. Singer, Proc. R. Soc. London, Ser. A 357, 37 (1977).

[15] J. P. Hansen and I. R. McDonald, Theory of Simple Liquids (Academic, London, 1986).

[16] G. Pastore, P. Ballone, and M. P. Tosi, J. Phys. C 19, 487 (1986).

[17] M. Parrinello and M. P. Tosi, Riv. Nuovo Cimento 2, No. 6 (1979).

[18] P. S. Salmon, Proc. R. Soc. London, Ser. A 437, 591 (1992); A. C. Barnes, S. B. Lague, P. S. Salmon, and H. E. Fisher, J. Phys.: Condens. Matter 9, 6159 (1997).

[19] O. Alcaraz, Ph.D. thesis, Universitat Politècnica de Catalunya (2000). 